\title{
Schwere Influenza: Was tun, was nicht?
}

\author{
Etwa 30\% aller Patienten, die wegen einer Influenza intensivstationär behandelt werden, \\ entwickeln schwere respiratorische Komplikationen. Wie lässt sich ihre Prognose verbessern?
}

Etwa 30\% aller Patienten, die wegen einer Influenza intensivstationär behandelt werden, entwickeln schwere respiratorische Komplikationen bis hin zum Lungenversagen (ARDS).

„Eine influenzaassoziierte Pneumonie kann sehr schnell fortschreiten“, warnte Prof. Marcos I. Restrepo von der Universität in San Antonio (Texas). Ein Patient, der mit einer Pneumonie aufgenommen werde, könne schon wenige Stunden später das Vollbild eines akuten Lungenversagens zeigen. Er riet dringend davon $\mathrm{ab}$, sich zur Prognoseabschätzung auf gebräuchliche Scores wie den PSI (Pneumonia Severity Index) zu verlassen. „Pneumoniespezifische Scores unterschätzen bei Influenza den Schweregrad der Erkrankung. Sie sollten nicht verwendet werden, um über intensivmedizinische Maßnahmen zu entscheiden.“

Wichtig ist, so Restrepo, dass „so früh wie möglich“ eine antivirale Therapie eingeleitet wird. Durch eine virostatische Therapie ab Tag 1 oder 2 nach Symptombeginn kann das Risiko für ARDS und Tod wahrscheinlich stärker reduziert werden als bei Therapiebeginn nach Tag 7; in einer Beobachtungsstudie wurden ARDS-Raten von $17 \%$ vs. $37 \%$ und eine Mortalität von $7 \%$ vs. $35 \%$ festgestellt. „Wenn der klinische Verdacht auf eine Influenza besteht, sollten Sie antiviral behandeln - selbst wenn der Schnelltest negativ ist", betonte der Pneumologe. Das Virostatikum der Wahl ist der Neuraminidasehemmer Oseltamivir. Ob eine Behandlungsdauer von fünf Tagen ausreicht, macht Restrepo bei Intensivpatienten von der RT-PCR abhängig: Wenn sie weiter positiv ist, setzt er die Behandlung fort.

Die "großen Probleme“ bei Influenzakranken sind Restrepo zufolge oft Konsequenzen einer bakteriellen Superinfektion. Einen ersten diagnostischen Hinweis auf eine bakterielle Beteiligung kann ein Procalcitonin-Test geben: Bei nicht immunsupprimierten Patienten sind erhöhte Werte ein Zeichen für eine bakterielle Pneumonie. Vor allem aber haben niedrige ProcalcitoninWerte bei Patienten ohne Schock einen hohen negativen Vorhersagewert. Restrepo räumte aber ein, dass er bei diesen Schwerkranken nicht den Mut habe, auf ein Antibiotikum zu verzichten, solange eine bakterielle Koinfektion nicht durch die Kultur ausgeschlossen sei. Die Hoffnung, dass ein Makrolid aufgrund seiner immunmodulatorischen Wirkung auch in Abwesenheit einer bakteriellen Pneumonie günstige Effekte hat, konnte nicht bestätigt werden. „Die Prognose wird dadurch nicht verbessert.“

Systemischen Kortikosteroiden wird ein möglicher Zusatznutzen bei stationären Pneumoniepatienten zugeschrieben. Bei der influenzaassoziierten Pneumonie ist das nicht der Fall. In Beob-

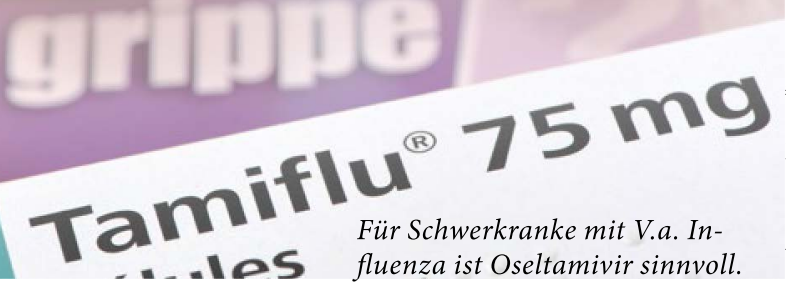

achtungsstudien wurde sogar ein Anstieg der Mortalität beobachtet. Dazu Restrepo: „Wenn Sie ihre Mutter behandeln, verwenden Sie keine Kortikosteroide! Wenn Sie Ihre Schwiegermutter behandeln, können Sie darüber nachdenken.“

Der Nutzen einer nicht invasiven Beatmung (NIV) bei Influenza-Pneumonie wird kontrovers diskutiert. Restrepo verzichtet meist darauf, solange nicht eine andere Indikation vorliegt, etwa eine akute Exazerbation einer COPD mit hyperkapnischem Versagen. Falls eine invasive Beatmung der Patienten nötig ist, sollte lungenprotektiv mit niedrigen Tidalvolumina beatmet werden.

Bei fortschreitendem ARDS kann zur Verbesserung der Oxygenierung die Umlagerung des Patienten vom Rücken auf den Bauch sinnvoll sein. Laut Restrepo können dadurch die Überlebenschancen verbessert werden. Bei schwerem ARDS müsse man außerdem die Gabe von Muskelrelaxantien in Erwägung ziehen. Die Therapie sei ebenfalls mit verminderter Mortalität assoziiert.

Nicht eingesetzt werden soll NO: „Die Inhalation von NO verbessert zwar die Oxygenierung, aber nicht das Überleben und kann nephrotoxisch wirken." Restrepo sprach sich auch gegen Hochfrequenzbeatmung (HFOV) aus. Bei schwerem ARDS werde die Sterblichkeit nicht reduziert, möglicherweise nehme sie gar zu. Wenn trotz Bauchlage und neuromuskulärer Blockade keine ausreichende Sauerstoffsättigung erreicht wird, kann die extrakorporale Membranoxygenierung (ECMO) in Betracht gezogen werden. Wegen der damit verbundenen Risiken sollte dies laut Restrepo aber nur in Krankenhäusern mit viel ECMO-Erfahrung geschehen. „Die ECMO ist riskant und teuer, aber bestimmten Patienten kann sie das Leben retten." Dr.BeateSchumacher

Symposium „Influenza and respiratory physicians", Kongress der European Respiratory Society, London, 3.-7. September 2016

\section{Aktive COPD-Patienten sind auch mental fitter}

COPD-Patienten senken ihr Risiko für Angsterkrankungen und Depression, wenn sie körperlich aktiv bleiben.

Regelmäßige Bewegung ist bei COPD ein essentieller Therapiebaustein, um die Abnahme der Lungenfunktion und den Muskelabbau zu mindern. Nun stellt sich heraus, dass sich dadurch auch das Risiko mentaler Komorbiditäten ein wenig mindern lässt, was deshalb relevant ist, weil bis zu 40\% der COPD-Patienten an Angstzuständen und Depressionen leiden.

Autoren aus den Niederlanden und der Schweiz hatte bei 409 COPDPatienten in Hausarztpraxen über fünf Jahre Korrelationen zwischen dem Ausmaß körperlicher Aktivität und dem Auftreten von Komorbiditäten untersucht. Patienten, die sich regelmäßig körperlich anstrengen, hatten ein um 11\% niedrigeres Angst- und um 15\% niedrigeres Depressionsrisiko hatten. Beziehungen zu anderen Komorbiditäten waren nicht signifikant. de

ERS-Kongress, London, 3.-7.9. 2016 